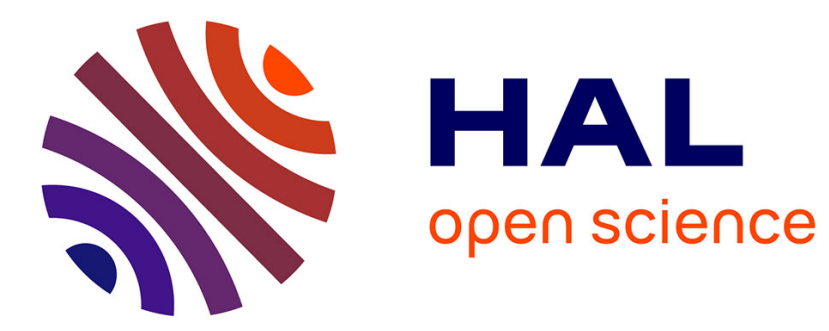

\title{
Controlled spontaneous emission of a tri(8-hydroxyquinoline) aluminum layer in a microcavity
}

B. Masenelli, A. Gagnaire, L. Berthelot, J. Tardy, J. Joseph

\section{To cite this version:}

B. Masenelli, A. Gagnaire, L. Berthelot, J. Tardy, J. Joseph. Controlled spontaneous emission of a tri(8-hydroxyquinoline) aluminum layer in a microcavity. Journal of Applied Physics, 1999, 85 (6), pp.3032-3037. 10.1063/1.369639 . hal-01807509

\section{HAL Id: hal-01807509 \\ https://hal.science/hal-01807509}

Submitted on 4 Jun 2018

HAL is a multi-disciplinary open access archive for the deposit and dissemination of scientific research documents, whether they are published or not. The documents may come from teaching and research institutions in France or abroad, or from public or private research centers.
L'archive ouverte pluridisciplinaire HAL, est destinée au dépôt et à la diffusion de documents scientifiques de niveau recherche, publiés ou non, émanant des établissements d'enseignement et de recherche français ou étrangers, des laboratoires publics ou privés. 


\title{
Controlled spontaneous emission of a tri(8-hydroxyquinoline) aluminum layer in a microcavity
}

\author{
B. Masenelli, A. Gagnaire, L. Berthelot, J. Tardy, and J. Joseph ${ }^{\text {a) }}$ \\ Laboratoire d'électronique, d'optoélectronique et microsystèmes, Unité associée au CNRS 5512, \\ Ecole Centrale de Lyon, 36 av. Guy de Collongue, BP 163, 69131 Ecully, France
}

(Received 14 October 1998; accepted for publication 17 December 1998)

\begin{abstract}
We report the fabrication of all-dielectric microcavities with a tri(8-hydroxyquinoline) aluminum $\left(\mathrm{Alq}_{3}\right)$ organic layer as the emitting layer. In a first step, we characterized the materials used in the structures by ellipsometry, and ensured nondegradation of the organic material in the fabrication process. Then, by angular-resolved photoluminescence, we investigated changes in the angular emission pattern caused by the cavities and observed a sharply directed emission. We also investigated the influence of the position of the radiative layer in the cavity on normal spontaneous emission. We observed enhancements in spontaneous emission over 20 times higher than that of a single $\mathrm{Alq}_{3}$ layer. These are the highest reported for organic material based microcavities. They are mainly explained by the very small thickness of the $\mathrm{Alq}_{3}$ layer $(20 \mathrm{~nm} \equiv 0.06 \lambda, \lambda$ being the resonant wavelength), by high-quality low-loss dielectric mirrors as well as by the narrow collecting angle of our experiment $\left( \pm 3^{\circ}\right)$. This study corroborates analogous works and demonstrates the possibility of controlling the spontaneous emission of an emitter by a microcavity. (C) 1999 American Institute of Physics. [S0021-8979(99)06406-3]
\end{abstract}

\section{INTRODUCTION}

The discovery of highly luminescent polymers ${ }^{1}$ and small evaporated organic molecules, such as $\operatorname{tri}(8-$ hydroxyquinoline) aluminum $\left(\mathrm{Alq}_{3}\right),{ }^{2}$ offered new opportunities in the field of luminescent devices. Their electroluminescence properties and high quantum efficiency are extensively exploited for the realization of light-emitting diodes $(\mathrm{LED})^{3-7}$ despite some major drawbacks such as their short lifetime. Their broad spectrum, spanning the visible range, allows easy tunability for the production of multicolor pixels. ${ }^{8}$ Moreover, some molecules and blends (dye-doped organic materials) even show more attractive properties such as a lasing effect. ${ }^{9-12}$ From a practical point of view, they offer a low-cost and relatively simple alternative to inorganic semiconductors which require expensive and timeconsuming deposition methods for the production of LEDs.

The optical properties of organic LEDs can be improved by the use of light-confining structures, i.e., microcavities. Like a filter, microcavities reduce the organic spectrum linewidth to the wavelength of the mode resonant along the normal axis. The intensity of this mode can be increased or decreased compared to the case of an organic layer without a microcavity, depending on cavity design.

Purcell ${ }^{13}$ first predicted the spontaneous emission modification of an emitter placed in a resonator. Extensive theoretical $^{14-19}$ and experimental works followed. ${ }^{8,20-23}$ It is now established that luminescence is the consequence of coupling between an emitter and a photon field at its location. The spontaneous emission rate of a dipole is described in the first-order perturbation theory by the Fermi golden rule:

\footnotetext{
${ }^{\text {a)} E l e c t r o n i c ~ m a i l: ~ j a c q u e s . j o s e p h @ e c-l y o n . f r ~}$
}

$$
\Gamma=\frac{2 \pi}{\hbar^{2}}|\langle f|d . E(r)| 0\rangle|^{2},
$$

where $d$ is the dipole moment, $E$ the electric field at the dipole location, and $\rho$ the electric field density of states; $|0\rangle$ and $|f\rangle$ represent the system with the dipole excited and no photon in the cavity and the system with the dipole deexcited and one photon in the cavity, respectively. This equation shows that by modifying the vacuum electric field at a dipole position, one can modify the spontaneous emission of this dipole.

Controlling the vacuum electric field can be achieved by the use of a resonator, i.e., a Fabry-Pérot cavity. A cavity modifies both the electric field and the density of states of the field at its location. The cavity mirrors introduce boundary conditions on their surface so that the projection of the wave vector of any lightwave existing in the cavity is a multiple of $\pi / L, L$ being the distance between the two mirrors:

$$
k \cos \theta=2 \pi n / \lambda \cos \theta=m \pi / L,
$$

where $n$ is the refractive index of the material filling the cavity, $\lambda$ the wavelength of the considered light, $\theta$ the angle between the wavevector $k$, and the axis normal to the cavity surface.

Consequently, any electric field existing in the cavity has a standing wave (sinusoidal) component along the axis normal to the cavity. Hence, one can control the spontaneous emission rate by controlling the position of the emitting layer in the cavity: placing it at a maximum of the field will drastically enhance radiation, while placing it at a node of the field will result in suppression of radiation. The other parameter to be controlled is the field density of states. This can be done through control of mirror reflectivity and cavity thick- 


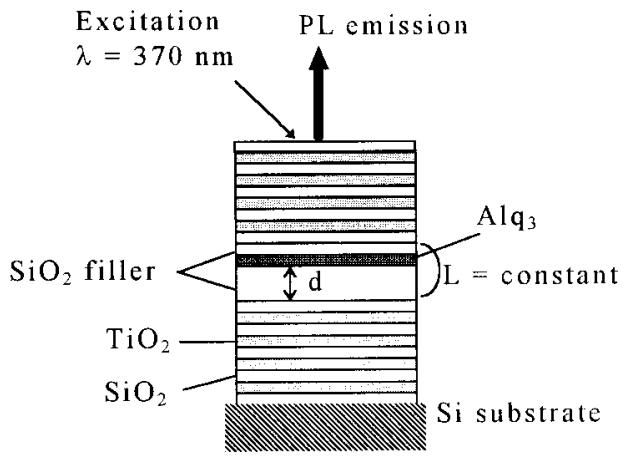

FIG. 1. Microcavity structure and geometry of the photoluminescence experiment.

ness $L$. High-reflectivity mirrors and small cavities (microcavities) are required to achieve a strong radiative emission.

This article reports on the photoluminescence study of organic material based microcavities with dielectric mirrors. So far, to our knowledge, all organic material based microcavities have been made with one metallic mirror. We show here the feasibility of microcavities with two dielectric Bragg reflectors. Moreover, great attention was paid to the control of layer thickness. In order to quantify the modification of emitted power according to the position of the emitting layer in the structure, we used a layer of tri(8hydroxyquinoline) aluminum $\left(\mathrm{Alq}_{3}\right)$ that was as thin as possible. Its optical thickness was $0.06 \lambda$, where $\lambda$ is the resonance wavelength along the normal to the cavity.

The structure and fabrication of the microcavities are detailed in Sec. II. In Sec. III, the optical characterizations of materials and structures are detailed. In particular, the results of photoluminescence experiments are reported. The angular dependence of the emission spectrum is first pointed out. Then, dependence of the power emitted along the direction normal to the cavity is shown. These results are confronted with theoretical calculations.

\section{MICROCAVITY DESIGN AND EXPERIMENTAL PROCESS}

Four microcavities, together with a noncavity control sample with a bare $\mathrm{Alq}_{3}$ layer on a silicon substrate, were made. Each structure was made of two distributed Bragg reflectors (DBR) surrounding a 20-nm-thick $\mathrm{Alq}_{3}$ emissive layer $(n=1.72$ at $\lambda=520 \mathrm{~nm}$ ). The position of the emissive layer, measured from the surface of the lower Bragg reflector and named $d$, was varied. Cavity thickness $L$ was held constant with one or two $\mathrm{SiO}_{2}$ filling layers (cf. Fig. 1).

The DBRs were designed to offer maximum reflectivity at $\lambda=520 \mathrm{~nm}$. Lower and upper DBRs consist of four and a half pairs of $\lambda / 4$ thickness $\mathrm{TiO}_{2}(n=2.1, t=62 \mathrm{~nm}) / \mathrm{SiO}_{2}(n$ $=1.43, t=91 \mathrm{~nm}$ ) layers. Silicon dioxide and titanium dioxide films were deposited at low temperature $\left(100^{\circ} \mathrm{C}\right)$ by electron cyclotron resonance (ECR) plasma-enhanced chemicalvapor deposition (PECVD) using, respectively, $\mathrm{O}_{2}$ and tetraethoxysilicate (TEOS) or titanium isopropoxide (TIPT) as precursors. $^{24}$

The thickness of each layer was controlled by means of an in situ ellipsometer. The evolution of the ellipsometric parameters $(\psi, \Delta)$ during the growth was compared to a theoretical prediction, coming from a custom-designed software. This enabled us to monitor the deposition of each layer accurately. The details of this method are described elsewhere. ${ }^{25}$ The $\mathrm{Alq}_{3}$ layers were deposited by thermal evaporation at $10^{-6}$ mbar pressure and at a rate of $1.5 \AA / \mathrm{s}$. The thickness of $\mathrm{Alq}_{3}$ was measured in situ by a quartz crystal monitor and checked with a stylus profiler and ellipsometric measurements. As the $\mathrm{Alq}_{3}$ layers are somewhat fragile, it was necessary to check that the $\mathrm{O}_{2}$ plasma combined with a deposition temperature of $100{ }^{\circ} \mathrm{C}$ did not modify the $\mathrm{Alq}_{3}$ layer. Indeed, $\mathrm{Alq}_{3}$ layers were kept unmodified after processing the upper DBR as assessed by photoluminescence tests. The use of an ECR plasma with low energy species is probably the reason for this inoccuity.

Control samples of both upper and lower DBRs were deposited on a Si substrate to measure their reflectivity spectra. Spectrophotometer experiments also provided the reflectivity spectra of the cavities.

Emissions from the cavities and the noncavity control sample were characterized by photoluminescence (PL) experiments. The wavelength of the excitation beam was adjusted for each cavity to the wavelength corresponding to a reflectivity minimum of the upper DBR between 360 and $380 \mathrm{~nm}$ (in the $\mathrm{Alq}_{3}$ absorption range). A Xe lamp coupled with a monochromator provided the adjustable wavelength excitation. The incidence angle was set to $45^{\circ}$ (cf. Fig. 1). For quantitative measurements, all $\mathrm{Alq}_{3}$ layers had to receive the same optical power. At this angle, the reflectors, which already offer minimum reflectivity at around $360 \mathrm{~nm}$, have no greater reflectivity than that generated by the index difference between air and $\mathrm{Alq}_{3}$. It was then only to ensure that the incident light power was kept constant from one sample to another.

The spectra presented here were obtained with the same exciting power. The photoluminescence (PL) signal was collected in air and at room temperature by an optical fiber placed on a turntable and sent to a monochromator equipped with a Si photodetector. The numerical aperture of the optical collecting system defines a photon collecting angle of $\pm 3^{\circ}$.

\section{RESULTS AND DISCUSSION}

\section{A. Optical characterization of materials}

In order to design and model the structures accurately, we needed to know the material optical properties, i.e., their refractive indices. The $\mathrm{SiO}_{2}$ index values used to design the structures correspond to a mix of standard $\mathrm{SiO}_{2}$ from literature $^{26}$ and $7 \%$ of air in the Bruggeman effective medium approximation. ${ }^{27}$ The index values of $\mathrm{TiO}_{2}$ and $\mathrm{Alq}_{3}$ were obtained from spectroscopic ellipsometry experiments, fitted with a model developed by Forouhi and Bloomer ${ }^{28}(\mathrm{~F}$. B. model). Our results corroborate ellipsometric experiments by Celii et al. on $\mathrm{Alq}_{3} .{ }^{29}$ To our knowledge, this is the first time this model has been applied to organic materials with satisfactory results. Figure 2 shows the resulting dispersion curves for $\mathrm{Alq}_{3}$. This model is based on the theory of twolevel system-damped transition. In this context, both real in- 


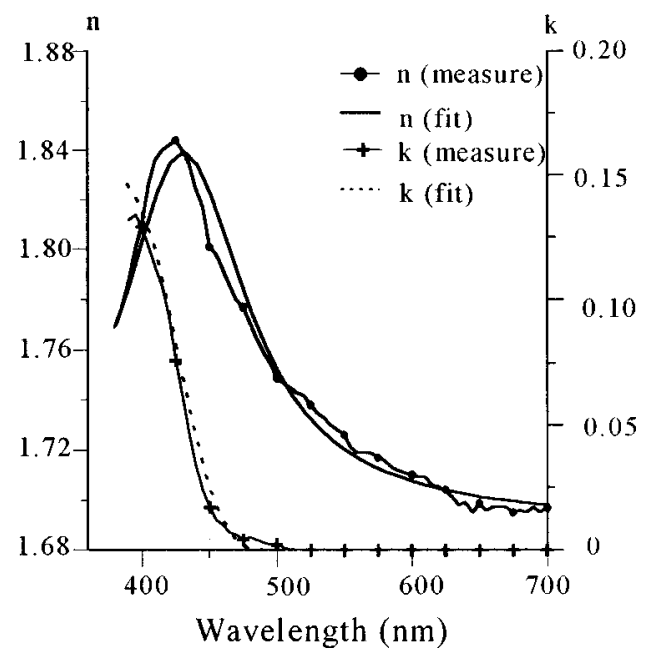

FIG. 2. Indices of refraction of $\mathrm{Alq}_{3}$ obtained from spectroscopic ellipsometry and fitted by the F. B. model

dex $n$ and imaginary index $k$ can be expressed as Lorentzian functions of energy transition $E_{t}$ in the following forms:

$$
\begin{aligned}
& k=\frac{A\left(E_{t}-E_{g}\right)^{2}}{E_{t}^{2}-B E_{t}+C}, \\
& n=n(\infty)+\frac{B_{0} E_{t}+C_{0}}{E_{t}^{2}-B E_{t}+C},
\end{aligned}
$$

where

$$
B_{0}=\frac{2 A}{\sqrt{4 C-B^{2}}}\left(-\frac{B^{2}}{2}+E_{g} B-E_{g}^{2}+C\right)
$$

and

$$
C_{0}=\frac{2 A}{\sqrt{4 C-B^{2}}}\left(\left(E_{g}^{2}+C\right) \frac{B}{2}-2 E_{g} C\right) .
$$

This model introduces five parameters: parameter A is related to the position matrix element of the transition; $\mathrm{B}$ is proportional to the middle-band energy difference; $\mathrm{C}$ is linked to the damping factor of the transition; $n(\infty)$ is the asymptotic value of the real index when $E_{t}$ tends to infinity and $E_{g}$ is band-gap energy. Table I sums up the values of these parameters obtained by a fit of the measured spectra for $\mathrm{TiO}_{2}$ and $\mathrm{Alq}_{3}$. The value of $\mathrm{Alq}_{3}$ band-gap energy is of particular interest. From Fig. 2, it can be considered as the minimum energy for which $k$ is not zero. The value of 2.54 $\mathrm{eV}$, obtained from the fit, is in fair agreement with the value of $2.7 \mathrm{eV}$ reported by Burrows et al. ${ }^{30}$

TABLE I. Parameters resulting from the fit of the ellipsometric measures by the F. B. model for $\mathrm{TiO}_{2}$ and $\mathrm{Alq}_{3}$.

\begin{tabular}{cccccc}
\hline \hline Material & \multicolumn{1}{c}{$\mathrm{A}$} & $\mathrm{B}$ & $\mathrm{C}$ & $n(\infty)$ & $E_{g}(\mathrm{eV})$ \\
\hline $\mathrm{TiO}_{2}$ & 0.46 & 7.41 & 0.91 & 1.96 & 3.08 \\
$\mathrm{Alq}_{3}$ & 0.088 & 5.71 & 0.14 & 1.69 & 2.54 \\
\hline \hline
\end{tabular}

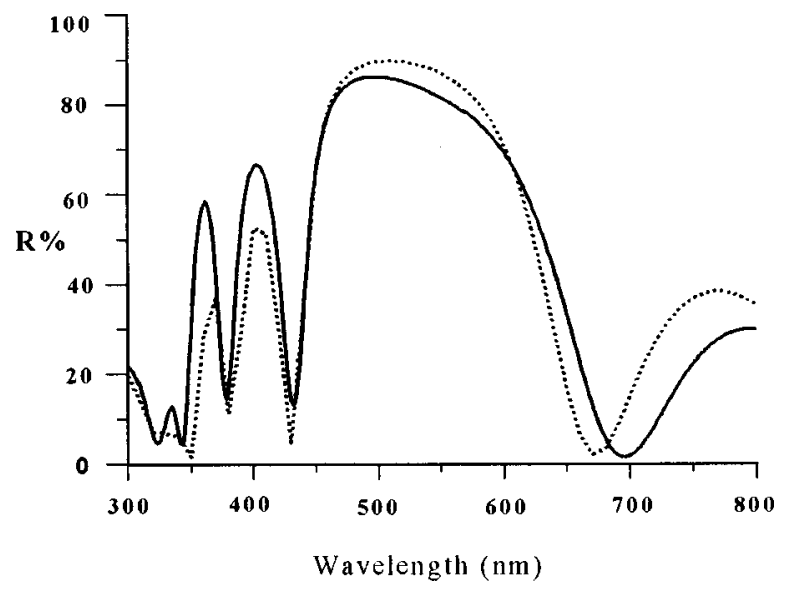

FIG. 3. Theoretical (dotted line) and experimental (solid line) reflectivity of the lower reflector control sample.

\section{B. Passive optical characterization of the structures}

Once material optical properties were known, the structures were made and characterized by spectrophotometer experiments (passive characterization). Figure 3 shows the spectrum of the lower reflector control sample. The results of its modeling using a transfer matrix method ${ }^{31}$ are also plotted for comparison. The spectrum exhibits a maximum of $86 \%$ reflection at $510 \mathrm{~nm}$ and a stop band of $100 \mathrm{~nm}$. The smaller stop band of the model compared to the experimental one results from the spatial inhomogeneity of layers: the size of the spectrophotometer beam spot being large $(8 \times 3 \mathrm{~mm})$, the collected signal is an average of signals from different zones whose spectra are slightly shifted. Despite this fact, consistency between the measured spectrum and the calculated spectrum is correct.

The upper reflector control sample exhibits a spectrum similar to that of the lower reflector control sample. However, it must be noted that the cavities defined by these DBRs are not symmetric. Whereas the reflectivity value of the lower reflector is truly identical to that of the control sample and attains $86 \%$, the reflectivity of the upper DBR is less than $86 \%$, given the fact that the last interface of the mirror is not $\mathrm{SiO}_{2} / \mathrm{Si}$ but $\mathrm{SiO}_{2} /$ air. This leads to upper mirror reflectivity attaining $70 \%$ instead of $86 \%$.

The theoretical and experimental reflectivity spectra of one microcavity are plotted in Fig. 4. Correlation between them is good. The experimental spectrum reaches a reflectivity minimum at $515 \mathrm{~nm}( \pm 5 \mathrm{~nm}$ from one cavity to the other) with a $13 \mathrm{~nm}$ full-width at half-maximum (FWHM) while our transfer matrix based model predicts $10 \mathrm{~nm}$. Good consistency between the calculated and measured spectra of the cavity, particularly at the resonant wavelength, confirms the accuracy of each previous DBR modelization. The slight discrepancy between the calculated and measured FWHMs can be explained by a small difference in optical indices between the deposited materials and the data used to model these and also a slight difference between experimental and theoretical thicknesses. 


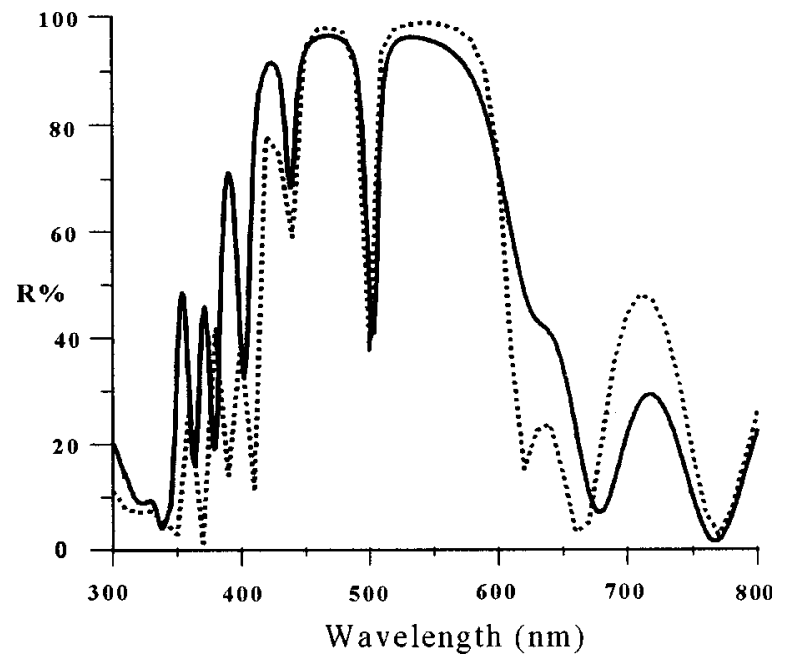

FIG. 4. Theoretical (dotted line) and experimental (solid line) reflectivity of a microcavity.

\section{Active optical characterization of the structures}

As mentioned in Sec. II, active characterization (i.e., dealing with the emission changes in the emitting layer) was performed by photoluminescence experiments. Figure 5 represents the variation of the resonant wavelength with the detection angle obtained from a microcavity with its emitting layer $45 \mathrm{~nm}$ from the lower DBR. As expected from the quantization equation of the wave vector normal component, the variation follows a cosine law [cf. Eq. (2)]. Figure 6 shows, for the same structure, the angle dependence of the $515 \mathrm{~nm}$ wavelength radiation [Fig. 6(a)] and of the $500 \mathrm{~nm}$ wavelength radiation [Fig. 6(b)]. In each case, the solid line is the result of a theoretical calculation and is normalized to the measured values. The angle reported in the figures is the angle inside the $\mathrm{SiO}_{2}$ cavity, which is related to the detection angle in air by the Snell-Descartes law. Our model is based on an "equivalent ideal metallic cavity." This means that

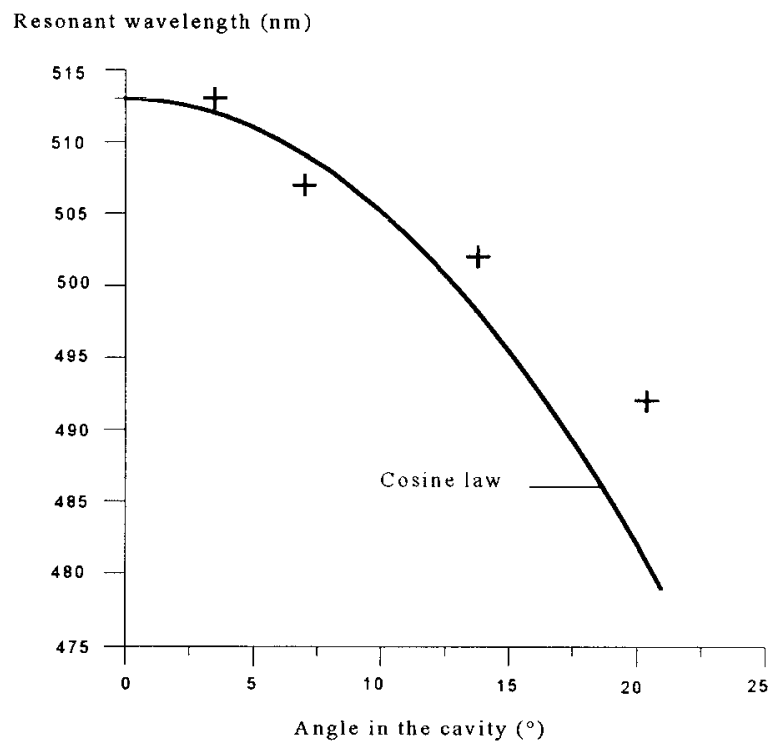

FIG. 5. Angle dependence of the resonant wavelength. instead of considering the whole real stack and performing an exact calculation, ${ }^{19}$ we consider a homogenous cavity with sharp reflecting interfaces, whose reflectivities are known, and in which lies the thin $\mathrm{Alq}_{3}$ emitting layer. ${ }^{16}$ The reflecting interfaces are separated by a distance $e$ so that:

$$
e=n L+2 l_{\text {pen }}=m \frac{\lambda}{2},
$$

where $L$ is the actual distance between the DBRs, $n$ the refractive index of the material in the cavity, $l_{\text {pen }}$ the penetration length of the light in the DBRs, $m$ an integer, and $\lambda$ the resonant wavelength along the structure normal axis. The penetration length in a Bragg reflector is obtained from the phase variation with a wavelength around that of maximum reflectivity $\left(\lambda_{\text {Bragg }}\right){ }^{16}$

$$
\left(\frac{d \phi}{d \lambda}\right)_{\lambda_{\mathrm{Bragg}}}=\frac{4 \pi n_{\mathrm{eff}} l_{\mathrm{pen}}}{\lambda^{2}},
$$

where $n_{\text {eff }}$ is the effective index of one period of the stack. According to this model, the penetration depth of the $\mathrm{TiO}_{2} / \mathrm{SiO}_{2}$ mirrors equals $3 \lambda / 4$, resulting in a cavity thickness of $4 \lambda / 2$, where $\lambda$ is approximately $515 \mathrm{~nm}$.

The $515 \mathrm{~nm}$ wavelength emission is resonant along the normal axis while the $500 \mathrm{~nm}$ is resonant at an angle of approximately $20^{\circ}$. The correlation between the predicted and measured angular diagrams is good for both wavelengths. We establish, as in previous work on analogous structures, ${ }^{21,22}$ that a microcavity concentrates every emission of a given wavelength on a particular cone.

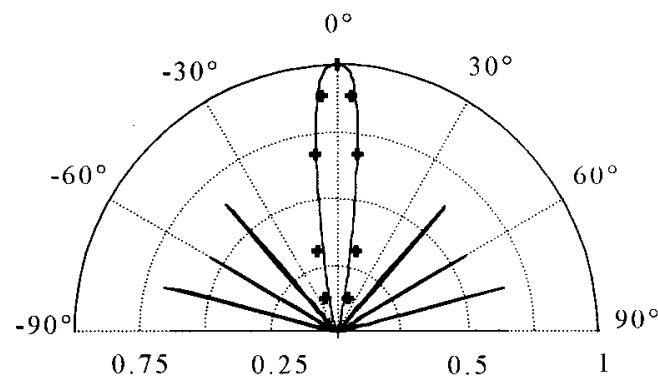

a)

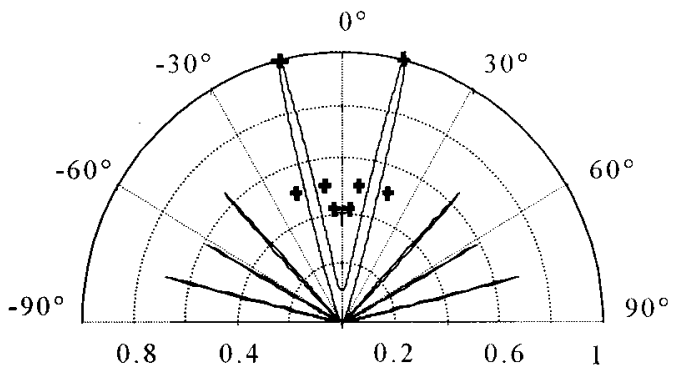

b)

FIG. 6. (a) Angular diagram of the emission at $515 \mathrm{~nm}$, (b) angular diagram of the emission at $500 \mathrm{~nm}$. The computed diagrams (solid lines) are normalized to the measured values $(+)$. 
The influence of the position of the $\mathrm{Alq}_{3}$ layer within the microcavity is shown in Fig. 7. Figure 7(a) shows the PL spectra of the cavities with the emitting layer 95 and $215 \mathrm{~nm}$ from the lower DBR along with the spectrum of a bare $\mathrm{Alq}_{3}$ layer of identical thickness (magnified 10 times for comparison). Figure 7(b) actually reports the ratio of the power emitted from each cavity along its normal axis at the corresponding resonant wavelength $(\lambda=515 \mathrm{~nm})$ to the power emitted under the same conditions by the noncavity control sample as a function of the position of the $\mathrm{Alq}_{3}$ layer from the lower DBR. The corresponding theoretical calculation is also plotted. According to the Fermi golden rule, the variation of this ratio is proportional to the square of the electric field intensity (the density of states being constant). The calculation was carried out assuming that the dipoles of the emitting layer are isotropically oriented.

Consistency between the model and the experiment is good for all the cavities but one. We have no definitive explanation for this deviation. One hypothesis is the photodegradation of the $\mathrm{Alq}_{3}$ layer of this sample since this cavity was

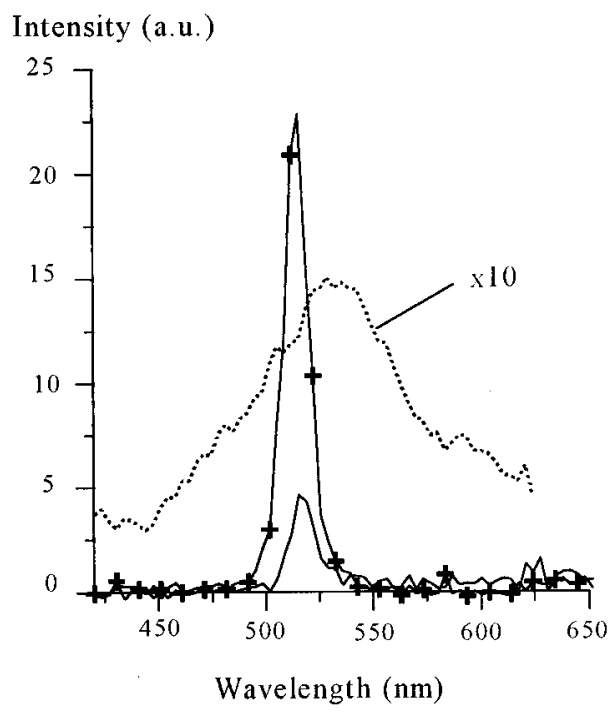

a)

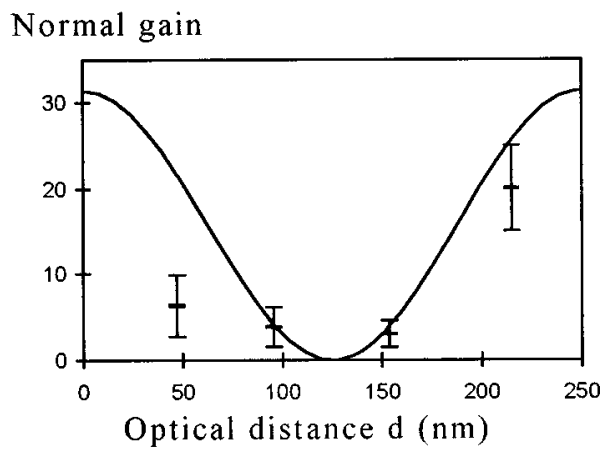

b)

FIG. 7. (a) PL spectra along the normal axis of a bare $\mathrm{Alq}_{3}$ layer $(\times 10$, dotted line), a cavity with its emitting layer $95 \mathrm{~nm}$ from the lower DBR (solid line), a cavity with its emitting layer $215 \mathrm{~nm}$ from the lower DBR (solid line with + symbols). (b) Variation of the normal gain at $515 \mathrm{~nm}$ as a function of the position $d$ of the emitting layer in the cavity. more widely used through our experiments. It should be pointed out that the normal spontaneous emission gain reported here for the cavity with its emitting layer placed at a field maximum is among the greatest ever published ${ }^{8,21,22,23}$ for an organic material based microcavity $(\approx 20)$. This results mainly from both the very thin $\mathrm{Alq}_{3}$ layer, which prevents the averaging of the coupling term $\left(|d . E(r)|^{2}\right)$ over a long distance, and the small collecting angle of the optical fiber.

\section{SUMMARY}

We have made organic material based microcavities, with all-dielectric reflectors and $\mathrm{Alq}_{3}$ as the emitting layer. This study reports the optical characterization of the materials and demonstrates the feasibility of such structures, and especially the nondegradation of the organic layer in a plasma-enhanced chemical vapor deposition reactor. These structures can be of interest for the production of microcavity devices emitting light from both sides. The only change from the structures presented here would be the use of a glass instead of a silicon substrate.

The microcavities demonstrate the same effects as dielectric/organic/metal microcavities. They enable control of the resonant wavelength and that of the angular emission pattern. Moreover, changing the position of the organic layer in the cavity results in modification of the normal spontaneous emission as is predicted by the Fermi golden rule. Our results are in agreement with other works dealing with analogous structures based on different materials. ${ }^{20,23}$ However, the results presented here are, to our knowledge, among the highest normal gains reported so far for organic material based microcavities. Consistency between the measurements and the model possibly shows that the effects of a microcavity on an organic layer can be predicted by a simple model.

${ }^{1}$ J. H. Burroughes, D. D. C. Bradley, A. R. Brown, R. N. Marks, K. Mackay, R. H. Friend, P. L. Burn, and A. B. Holmes, Nature (London) 347, 539 (1990).

${ }^{2}$ C. W. Tang and S. A. VanSlyke, Appl. Phys. Lett. 51, 913 (1987).

${ }^{3}$ D. O. Brien, A. Bleyer, D. G. Lidzey, D. D. C. Bradley, and T. Tsutsui, J. Appl. Phys. 82, 2662 (1997).

${ }^{4}$ G. Yu, C. Zhang, and A. J. Heeger, Appl. Phys. Lett. 64, 1540 (1994).

${ }^{5}$ C. C. Wu, J. C. Sturm, R. A. Register, and M. E. Thompson, Appl. Phys. Lett. 69, 3117 (1996).

${ }^{6}$ R. H. Jordan, A. Dodabalapur, M. Strukeljn, and T. M. Miller, Appl. Phys. Lett. 68, 1192 (1996).

${ }^{7}$ S. Noach, E. Z. Faraggi, G. Cohen, Y. Avny, R. Neumann, D. Davidov, and A. Lewis, Appl. Phys. Lett. 69, 3650 (1996).

${ }^{8}$ A. Dodabalapur, L. J. Rothnerg, R. H. Jordan, T. M. Miller, R. E. Slusher, and J. M. Phillips, J. Appl. Phys. 80, 6954 (1996).

${ }^{9}$ M. Berggren, A. Dodabalapur, and R. E. Slusher, Appl. Phys. Lett. 71, 2230 (1997)

${ }^{10}$ C. Zeng, W. Graupner, S. Tasch, G. Leising, K. Müller, and U. Scherf, Appl. Phys. Lett. 71, 2566 (1997).

${ }^{11}$ M. A. Diaz-Garcia, F. Hide, J. Schwartz, M. D. McGehee, M. R. Andersson, and A. J. Heeger, Appl. Phys. Lett. 70, 3191 (1997).

${ }^{12}$ G. J. Denton, N. Tessler, N. T. Harrison, and R. H. Friend, Phys. Rev. Lett. 78, 733 (1997).

${ }^{13}$ E. M. Purcell, Phys. Rev. 69, 681 (1946)

${ }^{14}$ D. Kleppner, Phys. Rev. Lett. 47, 233 (1981).

${ }^{15} \mathrm{~S}$. Haroche, course 13, in Les Houches, session LIII. 1990, Systèmes fondamentaux en Optique Quantique (unpublished), p. 967.

${ }^{16} \mathrm{G}$. Björk, Y. Yamamoto, and H. Heitmann, in Confined Electrons and Photons, New Physics and Applications, edited by C. Weisbuch and E. Burstein, NATO ASI Ser., Ser. B 340, 467.

${ }^{17}$ H. Yokoyama, Y. Nambu, and T. Kawakami, in Confined Electrons and 
Photons, New Physics and Applications, edited by C. Weisbuch and E. Burstein, NATO ASI Ser., Ser. B 340, 427.

${ }^{18}$ X. P. Feng and K. Ujihara, Phys. Rev. A 41, 2668 (1990).

${ }^{19}$ H. Rigneault and S. Monneret, Phys. Rev. A 54, 2356 (1996).

${ }^{20}$ N. E. J. Hunt, A. M. Vredenberg, E. F. Schubert, P. J. Becker, D. C. Jacobson, J. M. Poate, and G. J. Zydzik, in Confined Electrons and Photons, New Physics and Applications, edited by C. Weisbuch and E. Burstein, NATO ASI Ser., Ser. B 340, 715.

${ }^{21}$ T. Tsutsui, N. Takada, and S. Saito, Appl. Phys. Lett. 65, 1868 (1994).

${ }^{22}$ N. Takada, T. Tsutsui, and S. Saito, Appl. Phys. Lett. 63, 2032 (1993).

${ }^{23}$ D. G. Lidzey, D. D. C. Bradley, M. A. Pate, J. P. R. David, D. M. Whittaker, T. A. Fisher, and M. S. Skolnick, Appl. Phys. Lett. 71, 744 (1997).
${ }^{24}$ C. Martinet, V. Paillard, A. Gagnaire, and J. Joseph, J. Non-Cryst. Solids 216, 77 (1997).

${ }^{25}$ S. Callard, A. Gagnaire, M. P. Besland, and J. Joseph, Thin Solid Films 313/314, 479 (1998).

${ }^{26}$ E. D. Palik, Handbook of Optical Constants of Solids (Academic, New York, 1985).

${ }^{27}$ D. A. G. Bruggeman, Ann. Phys. (Leipzig) 24, 636 (1935).

${ }^{28}$ A. R. Forouhi and I. Bloomer, Phys. Rev. B 34, 7018 (1986).

${ }^{29}$ F. G. Celii, T. B. Harton, and O. F. Phillips, J. Electron. Mater. 26, 366 (1997).

${ }^{30}$ P. E. Burrows and S. R. Forrest, Appl. Phys. Lett. 64, 2285 (1994).

${ }^{31}$ M. Born and E. Wolf, Principles of Optics, 4th ed. (Pergamon, Oxford, 1970). 